\section{Regorafenib and the RESORCE trial: a new second-line option for hepatocellular carcinoma patients}

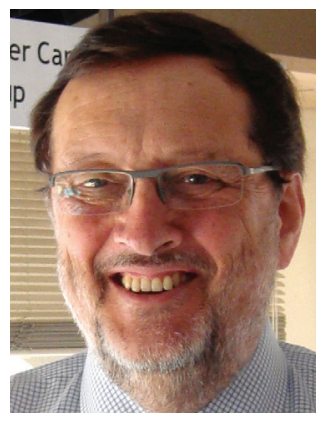

Dr Jordi Bruix* speaks to Laura Dormer, Editorial Director: Dr Jordi Bruix is the Head of the Barcelona Clinic Liver Cancer (BCLC) group at the Hospital Clinic (University of Barcelona, Spain) and Director of the Network for Research in Liver and Digestive Disease (CIBEREHD) in the Spanish Research Institute. His research has changed the diagnosis and treatment of liver cancer. Major highlights include: development of the BCLC staging and treatment; development of diagnostic criteria for hepatocellular carcinoma; identification of the value of portal pressure in predicting prognosis after resection; and acting as principal investigator of the Phase III trials that have shown the benefits of chemoembolization, sorafenib and regorafenib for patients with liver cancer. He has authored the European Assoaciation for the Study of the Liver, American Association for the Study of Liver Diseases and World Gastroenterology Organization Practice Guidelines and the United Network for Organ Sharing hepatocellular carcinoma (HCC) diagnostic criteria.

Accepted for publication: 11 July 2016; Published online: 19 August 2016

\section{Q What is the current standard of care for hepatocellular carcinoma patients? What are the main unmet needs for these patients?}

Treatment options for HCC patients include several approaches that have to be carefully selected according to tumor burden, liver function and physical status. The most widely used system to determine the first approach for treatment allocation is the BCLC model that combines all these factors. At early disease stages, patients may benefit from options such as surgical resection, liver transplantation or ablation. When tumors are multifocal and there is no vascular invasion or extrahepatic spread, and liver function and physical status are preserved, patients should be considered for chemoembolization. If these options are not feasible, patients should be considered for systemic treatment. In this setting, the sole agent that had been proven to improve survival through a large Phase III trial was sorafenib. Chemotherapy has not been shown to offer survival benefit and other agents that have been extensively evaluated have failed. The list of failed agents includes hormonal blockade, octreotide and several molecular-targeted agents with distinct profiles such as sunitinib, linifanib, brivanib, everolimus and ramucirumab. We have now shown in the RESORCE trial that we have an effective treatment - regorafenib - for second-line treatment after progression under sorafenib.

This is big news, but we still have several unmet needs for HCC patients. Recurrence is a frequent event after success with resection or ablation and we do not yet have any therapy

*BCLC Group, Liver Unit, Hospital Clinic, University of Barcelona, IDIBAPS, CIBEREHD, Villarroel 170, Barcelona 08036, Spain; Tel.: +34 93227 9803; Fax: +3493227 5792; jbruix@clinic.ub.es

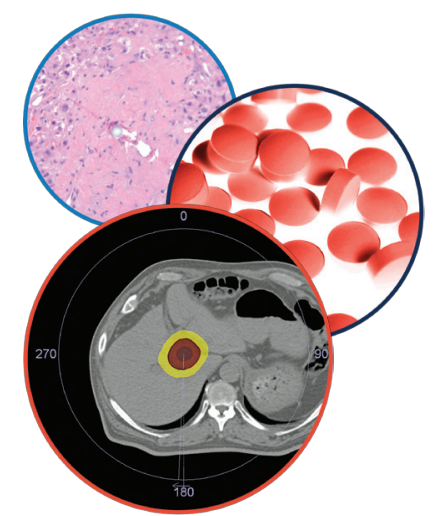

Hepatic Oncology

\section{KEYWORDS}

- hepatocellular carcinoma

- randomized controlled trial

- regorafenib • sorafenib • survival

- treatment 
that reduces such a risk. The same applies to an initial objective response with chemoembolization. HCC reactivates again during follow-up and it is important to develop a strategy to expand the duration of response. Indeed, one of the major challenges at present is to ensure that patients transition properly from chemoembolization into systemic therapy. Frequently, such locoregional procedures are repeated too many times even in the absence of response and this is associated with uncontrolled tumor progression and impairment of liver function. As a result, patients do not benefit from timely entry into systemic therapy and its associated survival improvement. Now we have sorafenib and regorafenib, but we need more effective agents to expand the current treatment benefits.

\section{Q What were the main aims of the RESORCE trial?}

The RESORCE trial assessed if regorafenib would improve survival of patients with HCC who had progressed under sorafenib. To clearly capture the potential efficacy of the drug, we carefully defined the target population. Patients should have confirmed HCC and confirmed progression under sorafenib, but at the same time their liver function should be preserved (Child-Pugh A) and their physical status should fit into performance status 0 or 1 . Patients were randomized in two arms comparing regorafenib at the standard dose of $160 \mathrm{mg} /$ day orally, 3 weeks on/1 week off versus placebo in a doubleblind fashion. Hence, the trial was designed to obtain the highest degree of scientific strength.

\section{Q What were the key findings of the trial?}

The main findings of the trial are that regorafenib is effective in improving survival and its administration is safe. Overall median survival was increased from 7.8 to 10.6 months, which represents a reduction in the risk of death of almost $40 \%$. This represents a very significant and clinically meaningful benefit that has surpassed the initial assumptions we had for the drug. The clear-cut benefit in survival was paralleled by a more than $50 \%$ reduction in progression-free survival and of tumor progression rate. Objective response rate, disease stabilization and duration of response were also significantly positive in the regorafenib arm. Furthermore, when we conducted subgroup analysis according to several parameters we observed that the positive effects of the drug were consistent across all categories and in all outcome parameters.

\section{Q How does the safety profile of regorafenib compare with sorafenib?}

The safety of sorafenib in HCC patients was optimal. Patients had the expected adverse events that are characteristic of multikinase inhibitors, including hand-foot skin reaction, arterial hypertension, fatigue and diarrhea. However, in most cases it was easy to manage with proper advice and dose adjustments. Only $10 \%$ of patients had to interrupt therapy because of drug-related adverse events. It is important to note that at the start of the trial we were concerned about potential intolerance, as this is a common perception by oncologists who have used the agent in patients with advanced colorectal cancer. Drug-related liver failure was suggested in some such patients, but in our study we only observed two such events and corresponded to the placebo arm. It is likely that a key difference between these separate populations is that HCC patients receive regorafenib as a second-line treatment after sorafenib, so that they are familiar with the adverse events profile. Contrarily, colorectal patients receive regorafenib after several lines of therapy with distinct toxicity profiles that may even persist at the time of regorafenib initiation.

\section{Q What are the overall implications of these findings for the management of HCC?}

The results of RESORCE will curb the pessimistic status of the field of systemic therapy for HCC. The series of failures that the field had experienced with several drugs magnified the concept of HCC being a very difficult to treat cancer, in which it was too risky to engage in the development of new agents. It was also said that the only way to be successful was to select the patients to be treated with novel agents according to their molecular profile, as identified by tumor biopsy sampling. However, everybody now accepts that tumors are heterogeneous and that a biopsy may offer unreliable information. Furthermore, we still need to demonstrate in prospective studies that any of the molecular signatures that have been proposed have a predictive value for any robust outcome parameter. The tissue analysis of the STORM trial testing if sorafenib would be effective in reducing recurrence after resection or ablation will offer such information, and inform 
about the validity of any of the messages that have been raised during recent years about personalized medicine for HCC according to molecular profile. I think that we will have to start again from time zero in such a field, but we will need to wait for our final analysis.

Q What are the next steps for the research in this area?

We need to understand better the meaning of the molecular abnormalities that have been described by all sorts of technologies. It is key to avoid a wishful thinking trying to link any of the observed patterns with a potentially effective target by any of the available agents. Genomic abnormalities may not be functionally active and what is a signal in cell cultures or experimental models may not be followed by a suggestive signal in humans. Indeed, one of the major needs in HCC and in cancer in general is to refine the methods to detect promising drug activity so that we leave behind the current definitions of RECIST and its variants.

Probably, the next challenge is to envision which agents should be combined with sorafenib or regorafenib, and also how to combine and schedule all of them. We have promising agents that are undergoing assessment in Phase III trials, such as tivantinib. This agent is being tested in patients with increased c-met expression in the tumor and hence, has a molecular enrichment that makes it appealing. At the same time, everybody is very optimistic about immunotherapy and such an approach has a positive 'aura'. However, 'aura' is not evidence-based and we need to have long-term data of all the studies that are ongoing.

Q Do you have any final comments for the readers of Hepatic Oncology?

In the RESORCE trial, we have shown that success is within reach. This is feasible if the drug is active and safe, the design of the trial is optimal and its execution pristine. I have been fortunate to be the principal investigator of RESORCE and 10 years ago of SHARP that proved the benefits of sorafenib. Two big highlights in a research career are a privilege and I am happy for the patients and for the success that all these results represent for the BCLC group that I have directed since its creation.

\section{Disclaimer}

The opinions expressed in this interview are those of the interviewee and do not necessarily reflect the views of Future Medicine Ltd.

Financial \& competing interests disclosure $J$ Bruix has received consulting and grant support from Bayer Healthcare.J Bruix has also received consulting from the following: Bayer, Arqule, Kowa, Lilly, Bristol-Myers Squibb, Merck, Terumo, BTG, Syrlex, Roche, Medimmune, Novartis and Onxeo. The author has no other relevant affiliations or financial involvement with any organization or entity with a financial interest in or financial conflict with the subject matter or materials discussed in the manuscript apart from those disclosed.

No writing assistance was utilized in the production of this manuscript. 